\title{
AC 2009-2352: THE “BOX METHOD” FOR TEACHING RATIO/PROPORTION PROBLEMS
}

James Sullivan, Dallas Independent School District 


\title{
The "Box Method" for Teaching Ratio/Proportion Problems
}

\begin{abstract}
This paper details a systematic method for teaching high school students how to set up and solve ratio and/or proportion problems. Such problems frequently occur in a wide variety of engineering applications. The author, while teaching high school algebra courses, noticed a remarkable fact: Students were able to solve such problems correctly once the problems had been set up properly. In other words, their major difficulty was not in the arithmetic required to solve these types of problems, but simply in setting the problems up. After examining several textbooks, the problem became clearer: this important aspect of solving ratio/proportion problems has been neglected for many years.
\end{abstract}

The author theorized that this learning, as most other learning, takes place in very small "microsteps". Teachers are familiar with the solution to such problems and tend to gloss over the essential phase of setting up such problems. As indicated above, algebra textbooks also neglect this important aspect of solving such problems. Students need structure while they are learning this type of process, and this fact has been overlooked for too long in the pedagogy of such problems.

The author then developed a highly-structured, systematic means of setting up such problems. Students quickly began to set up the problems correctly. Once students had the problems set up correctly, completing the arithmetic details of the solution was easy for them. The result was an almost perfect success rate for students working on ratio/proportion problems. A few simple math errors remain, but student success rates have been dramatically improved using this method.

This paper details the "box method" and how it should be taught. Several examples are provided to illustrate the use of this method.

\section{Introduction}

Ratio/proportion problems are a key area of mathematics often used in science, engineering, and business. Conversions of any type of linear direct variation, such as feet to inches, pounds to ounces, etc., are essentially ratio/proportion problems. Very often, similar triangles provide opportunities for using ratio/proportion analysis to determine the unknown lengths of the sides of these triangles. And maps, blueprints, and photographs often serve as the basis for ratio/proportion problems.

The arithmetic involved is usually minimal. In its simplest form, only one multiplication and one division are required. Yet students frequently have serious difficulties learning how to use such a fundamental tool. In fact, a 2007 report on standardized testing of mathematics found that when attempting to master standards for 8th grade coordinate geometry, "Students who are unsuccessful have the greatest difficulty with setting up and solving proportions from real-world 
examples involving similar triangles" (in addition to three other factors). ${ }^{1}$ Furthermore, one of the report's recommendations is "For Grades 6-8, students need more experience setting up and solving proportions from problems presented in a real-world context." 2 And with respect to the Florida Comprehensive Assessment Test (FCAT) $10^{\text {th }}$ grade level standard of understanding mathematical operations, "Students who are successful are able to ... understand and apply proportion concepts" (in addition to other skills). ${ }^{3}$ Finally, ratio/proportion skills are important to the successful performance of the FCAT $7^{\text {th }}$ grade standard of measurement, especially concerning interpreting scale drawings. ${ }^{4}$ There is every reason to believe that these findings apply not just to Florida students, but also to students everywhere.

The author discovered that the root cause of so many students having trouble understanding how to solve these types of problems is that some "micro-steps", (which most teachers learned once and forgot) are not communicated to students in a structured format. When students are taught the forgotten micro-steps in a structured format, the result is an almost $100 \%$ success rate in setting up and solving ratio/proportion problems.

In this paper, first we will review an important concept that led to the development of this method. This may seem like a brief digression, but it is critical to many such problem areas in learning. Here it is in a nutshell: Never ignore the obvious! We review this principle because it may aid other teachers when developing strategies to help students learn other traditionally difficult topics.

Next, we use this principle to show how the "box method" was developed. Then several examples are provided to illustrate its use. Finally, we conclude this paper.

\section{What Experts Have Forgotten}

Before proceeding to the box method, let us review how this method evolved. To do so, we must first examine a seemingly unrelated idea: What is the cause of two car accidents?

Two cars, of course! Well, that's obvious, isn't it? So, what benefit does it provide us to know that?

The fact of the matter is that, in this case, the obvious leads us to techniques that can prevent accidents. Unless two cars are near each other, they cannot be involved in a two-car accident. It is simply impossible. So, in each incidence in which two cars can be in close proximity to one another, care must be used and appropriate strategies must be developed. For instance, whenever possible, we should drive so as stay as far away as practical from other vehicles.

Many years ago, Shell Oil Company published a series of pamphlets called the Shell Answer Book Series. In one of those booklets, a professional driver provided his insights. Drive with a "shield of vacant traffic" surrounding you. When cars get close to you in traffic, either slow down, speed up, or change lanes so as to move into another "shield" in which there are no cars in close proximity to you. That's not always possible, but when you can, you know you won't be hit from anyone around you, simply because there is no one in your protective "bubble". 
Although the exact Shell Answer Book referenced above is no longer in print, here is a similar idea from one of the other books in the series: "Try not to let yourself become 'boxed' in. Instead, create a safe driving space around your car, leaving an 'open door' should someone suddenly slam on the brakes or make a sharp turn." 5

You might be tempted to think that scientists never ignore the obvious. Yet until Sir Isaac Newton formulated the law of gravity, no one apparently had thought about it enough to seriously ask the simple question, "Why do things fall?" The popular story that Newton was sitting under an apple tree when one fell off and hit him in the head may not be $100 \%$ accurate, but even Newton did admit his original inspiration to examine the phenomena of falling objects led him to formulate the law of gravity. ${ }^{6}$ Newton didn't ignore the obvious, and this led to an extremely important discovery, a discovery that had been delayed for centuries simply because people ignored the obvious: Objects fall.

It's a simple concept, but it all begins with the realization that the obvious must be explored for all that we can harvest from it.

Now, how do we apply this principle to ratio/proportion problems? When the author began examining why so many students were having problems with ratio/proportion problems, something immediately became obvious: The student errors were almost always due to setting the problems up incorrectly. The arithmetic was fine in almost every case. So, if they could learn how to do set up the problems correctly, the remaining arithmetic would be easy for them.

But why didn't the students understand how to set the problems up correctly? When someone first learns something, there is a focus on the details. As we become better and better at any new skill, we tend to perform more and more of the skill automatically. This is great when we are in the role of learners, but it is terrible for us when we are in the role of teachers. In essence, we have forgotten the details we need to solve the problem! We don't concentrate on the details after we learn something well, because we don't need to anymore. We carry out the details automatically. But to teach someone, we need to go back go the basics and proceed slowly through all of the details until the student catches on.

Many others have reached the above general conclusion. For instance, according to a 1999 report, "Experts are able to flexibly retrieve important aspects of their knowledge with little attentional effort." 7

And immediately following the above insight is this: "Though experts know their disciplines thoroughly, this does not guarantee that they are able to teach others." 8 This revelation should shock no one. Many of us have had teachers who knew the subject well, but did not know how to communicate it. They know how to do something; they just don't know how to tell someone else how to do it.

In fact, there is an old Chinese saying, "To teach is to learn twice." The first time is to learn how to do something, and the second time is to learn how to tell others how to do it. 
In many ways, the human brain can be, and often has been in the past, compared to a computer. When a computer is programmed, the hardware has to be given very simple, extremely basic instructions so that the computer does what it is supposed to do. In the author's experience as a teacher, this is true of humans as well. Things must be broken down into small digestible pieces so that the human mind can assimilate the parts and absorb the information properly. As we get better and better at any given task, this process can be sped up considerably. Of course, there are often intuitive leaps of understanding. But much of what we learn is through rote practice.

Although some believe that rote learning does not contribute to the development of understanding,

You can't understand what you don't remember. Knowledge must be stored in the brain. Practicing is one way to secure knowledge in memory. Once there, understanding can begin to develop. Each higher level of understanding typically results from new information stored in the brain. "Understanding" is a complex, poorly understood process that involves linking multiple stored "chunks" of knowledge. We have no idea how this magical process occurs. Sometimes we have the experience of the "light bulb turning on", but more frequently understanding gradually improves as new knowledge is added to the constantly growing remembered knowledge base in the brain. ${ }^{9}$

And here's what mathematician Keith Devlin, Executive Director of the Center for the Study of Language and Information at Stanford University, has to say about learning new skills:

When we learn a new skill, initially we simply follow the rules in a mechanical fashion. Then, with practice, we gradually become better, and as our performance improves, our understanding grows. Think, for example, of the progression involved in learning to play chess, to play tennis, to ski, to drive a car, to play a musical instrument, to play a video game, etc. We start by following rules in a fairly mechanical fashion. Then, after a while, we are able to follow the rules proficiently. Then, some time later, we are able to apply the rules automatically and fluently. And eventually we achieve mastery and understanding. The same progression works for mathematics, only in this case, as mathematics is constructed and carried out using our language capacity, the initial rule-following stuff is primarily cognitive-linguistic. ${ }^{10}$

Two items are very important consequences of the above insights. First, if we learn a skill the wrong way, it can be difficult to learn how to perform the skill correctly, since a large amount of "unlearning" may need to occur. Second, as pointed out previously, when students reach the "automatic" stage, they often have consciously forgotten the details of how the process is done. So if and when these students become teachers, they no longer focus on the details that new students' need to understand the process. Teachers may try to illustrate the process, but essential details are not provided.

And hence practice does not make perfect unless the practice is done properly. So, a method needed to be established that would allow students to collect the data from a ratio/proportion 
problem and then develop a correct equation. First, let us look deeper at the details most experts often forget when teaching ratio/portion problems. Then we can put the procedure into a welldefined, yet simple structure.

\section{A Deeper Analysis of Ratio/Proportion Problems}

What information does a ratio/proportion problem have to provide us? First of all, any ratio/proportion problem is a comparison of at least two items. More than two objects could be involved; for example, let's suppose a certain cake recipe required two eggs, 3 cups of milk, and 4 teaspoons of sugar. To make larger or smaller cakes, or to make fewer or more cakes, we will need to keep the quantities of these three items in proportion. However, any ratio/portion problem can be solved by comparing only two of the objects at a time. So, without loss of generality, we can presume that all such problems involve only the comparison of two items at any given time. In the cake example, we could first work the problem using just the eggs and the milk; then we might decide to complete the solution by using only the eggs and the sugar.

In any problem, by definition, there must be an unknown. For any ratio/proportion problem, we must have at least three other known quantities. So, our first step should be to identity the unknown item. In a word problem, the missing quantity will usually appear in the last sentence of the problem.

To be more precise, we will always have two known items, one of which will have one known and one unknown characteristic; the corresponding characteristics of the other item must both be known.

Now, let us proceed to put these insights into a structured problem-solving system. At the same time, we will use an example to illustrate how this would be carried out in practice.

\section{Application of the Insights Using Several Examples}

A series of examples will now be given to illustrate this process. Progressively less and less detail will be given in much the same way as students would be taught this process. Of course, students would be required to provide full details ("show all of your work") until they have achieved a satisfactory level of performance.

Example 1: A large scalene triangle and a small triangle are similar. The shortest sides of the triangles are 3 inches and 6 inches, respectively. The length of the longest side of the larger triangle is 10 inches. What is the length of the longest side of the smaller triangle?

From the last sentence, this time in the form of a question, we know that the unknown quantity is the length of the longest side of the smaller triangle. Let us call this "X" and draw a square containing three rows and three columns:

\begin{tabular}{|l|l|l|}
\hline & & \\
\hline & & \\
\hline & & \\
\hline
\end{tabular}


Put the " $\mathrm{X}$ " in the second column, second row, in much the same way as starting a game of tictac-toe.

\begin{tabular}{|l|l|l|}
\hline & & \\
\hline & $\mathrm{X}$ & \\
\hline & & \\
\hline
\end{tabular}

The second row could either be labeled as "the long side" or "the small triangle"; but whatever you decide to label this row, you need to label the second column by the other term. Note that what we are comparing in this problem is the sides of two different triangles; the compared objects will always give us the either the titles of the columns or the rows, depending on how we choose to label the rows and the columns of the box.

In fact, it doesn't really matter, in most instances, whether you label the second row or the second column as one of the compared items, as long as you are consistent. Arbitrarily, let's label the second row as "the long side" and the second column as "the small triangle".

\begin{tabular}{|l|l|l|}
\hline & the small triangle & \\
\hline the long side & $\mathrm{X}$ & \\
\hline & & \\
\hline
\end{tabular}

If the second column is "the small triangle", then the third column must be labeled as "the large triangle"; that is, whatever object the object in the first column is being compared to.

\begin{tabular}{|l|l|l|}
\hline & the small triangle & the large triangle \\
\hline the long side & $\mathrm{X}$ & \\
\hline & & \\
\hline
\end{tabular}

The problem tells us that the length of the long side of the large triangle is 10 inches. Let us insert this into our table now:

\begin{tabular}{|l|l|l|}
\hline & the small triangle & the large triangle \\
\hline the long side & $\mathrm{X}$ & 10 inches \\
\hline & & \\
\hline
\end{tabular}

Since the second row is titled "the long side", we need to label the third row as "the short side"; that is, whatever characteristics the long side is being compared to.

\begin{tabular}{|l|l|l|}
\hline & the small triangle & the large triangle \\
\hline the long side & $\mathrm{X}$ & 10 inches \\
\hline the short side & & \\
\hline
\end{tabular}


Now, let us fill in the given lengths of the triangles from the problem above:

\begin{tabular}{|l|l|l|}
\hline & the small triangle & the large triangle \\
\hline the long side & $\mathrm{X}$ & 10 inches \\
\hline the short side & 3 inches & 6 inches \\
\hline
\end{tabular}

We can now rewrite the above information as an equation:

$\mathrm{X} /(3$ inches $)=(10$ inches $) /(6$ inches $)$

Note that if the above had been written in pencil, we could simply erase the box and put an equal sign between the second and third columns of the data. We would then be ready to solve the problem arithmetically.

We can cancel out the units (in this case, inches) on the right-hand side of the equation, leaving

$\mathrm{X} / 3$ inches $=10 / 6$

If we multiply both sides by 3 inches, we have

$(\mathrm{X} / 3$ inches $) *(3$ inches $)=(10 / 6) *(3$ inches $)$

and simplifying,

$X=(10 / 2)$ inches $=5$ inches

Of course, as a concluding step to any math problem, it is always a good idea to check the final answer to make sure it truly is a solution. And note, it is very important to maintain the units (in this case, inches) in the problem. Often, different units may be used, and in such cases, conversions must be carried out to make sure all of the units are the same; we should neither be adding apples and oranges nor multiplying them! And it is interesting to recognize that measurement conversions are also ratio/proportion problems.

Since we have intentionally placed the unknown in the middle square, we avoid the additional step involved in cross-multiplication (also referred to as the "butterfly method'). In essence, the arithmetic needed to solve the problem, once it has been set up properly, is simply one multiplication and one division.

In summary, the box method is carried out using the following steps: Draw a 3 by 3 box, much like a tic-tac-toe board. Put an "X" in the center square. Label the second column and the second row with the descriptive information about the unknown (the name of the object and the compared characteristics of the object). Although it does not matter whether the object titles are in the rows or the columns, for the sake of consistency, arbitrarily label the second column with the object's title, and label the second row with the object's characteristic. The unknown quantity is usually indicated in the last sentence of the problem, so it is generally very easy to determine what is being sought in any given problem. 
Label the third column with the name of the other object in the problem. Label the third row with the other characteristic in the problem (in other words, the characteristic of the second object). Convert the numeric portion of the table to an equation by either (1) erasing lines and putting an equal sign in between the second and third columns, or (2) by copying the numeric information and inserting an equal sign in between the numbers. After that, completing the solution of the problem is straightforward, as shown above.

Example 2: A tree casts a shadow of 20 feet at the same time that a yardstick casts a 2-foot shadow. How tall is the tree?

First, convert the length of the yardstick to feet. One yard equals three feet. Note that we could also work this problem using yards, but since more of the measurements are in feet, converting yards to feet will require less effort than converting feet to yards for this problem.

The unknown is the height of the tree. (Note that we are dealing with 2 similar triangles again in this classic problem.) The items are the tree and the yardstick. The characteristics are the actual heights of the objects and the lengths of the shadows. Filling those in the box below, we obtain:

\begin{tabular}{|l|l|l|}
\hline & tree & Yardstick \\
\hline height & $\mathrm{X}$ & $3 \mathrm{feet}$ \\
\hline length of shadow & 20 feet & 2 feet \\
\hline
\end{tabular}

Having set up the problem properly, we switch to equation format:

$\mathrm{X} / 20$ feet $=3$ feet $/ 2$ feet $=3 / 2$

and then we multiply both sides by the denominator of the left-hand side of the equation:

$\mathrm{X}=(3 / 2) * 20$ feet $=30$ feet

Example 3. A certain cake recipe requires 3 eggs and 4 cups of milk per cake. If you have 9 eggs, how many cups of milk do you need to cook the maximum number of cakes with the 9 eggs?

Unknown: cups of milk to use up all the eggs

Objects: milk and eggs

Characteristics: the number needed to use all supplies up and the number needed for one cake

\begin{tabular}{|l|l|l|}
\hline & milk (in cups) & Eggs \\
\hline $\begin{array}{l}\text { the number needed to use } \\
\text { up all supplies }\end{array}$ & X & 9 \\
\hline $\begin{array}{l}\text { the number needed to make } \\
\text { one cake }\end{array}$ & 4 & 3 \\
\hline
\end{tabular}


$\mathrm{X} / 4=9 / 3=3$

so

$\mathrm{X} / 4=3$

Multiply both sides by 4 to eliminate the denominator on the left-hand side:

$(\mathrm{X} / 4) * 4=3 * 4=12$

$\mathrm{X} / 1=\mathrm{X}=12$

Note that the units above would all cancel out: cups over cups on the left hand side of the equation, and eggs over eggs on the right-hand side of the equation.

In the next three examples, we identify the unknown item, the objects compared, and the compared characteristics of the objects. After that, the reader should easily be able to "fill in the box" and complete the solution to the problem. By presenting this in the form of a "tic-tac-toe" board, students perceive this as a game and become even more engaged in the solution steps.

Example 4. The distance on a Texas map from Dallas to Austin is 4 inches. If the map scale is 1 inch $=50$ miles, how far is it from Dallas to Austin?

Unknown: actual distance from Dallas to Austin

Compared objects: map and the real world

Compared characteristics: distance on map and actual distance

Example 5: A photograph is 3 inches by 5 inches. If the photograph is enlarged so that the long side is 8 inches, how wide will the photograph be?

Unknown: the width of the enlarged photo

Compared Objects: original photo and the enlarged photo

Characteristics: the length and the width of the photos

Example 6: On a blueprint, the height of a room is 16 centimeters. If the scale of the blueprint is $2 \mathrm{~cm}=1$ meter, how tall will the actual room be?

Unknown: the height of the actual room

Objects: blueprint and the actual room

Characteristics: height on the blueprint and height in the real world

By now, the reader should have a very solid understanding of this technique and should be well prepared to teach this technique to students. 


\section{Conclusion}

This box method is an effective tool for teaching students how to successfully solve ratio/proportion problems. It provides the additional detail and structure required for students to understand how to set these problems up correctly. After that, completing the problem involves only one multiplication and one division.

But beyond simply teaching students how to solve ratio/proportion problems, teachers should ask themselves, whenever students are having problems learning something new, what additional details are needed for students to completely understand the process? What things are "obvious" to the teacher, but not the students? What details has the teacher forgotten that students need to know? Once any process is understood on this level, it can be more effectively communicated to students. And perhaps new techniques for teaching can be shared as similar teaching tools are developed for other classes of math problems

\footnotetext{
${ }^{1}$ FCAT (Florida Comprehensive Assessment Test) Mathematics Lessons Learned. Florida Department of Education. 2007 [cited 5 February 2009]. Page 122. Available online as of February 5, 2008 at: http://fcat.fldoe.org/pdf/FCAT07_LL_Math.pdf

${ }^{2}$ Ibid, page 123 .

${ }^{3}$ Ibid, page 85 .

${ }^{4}$ Ibid, page 97.

${ }^{5}$ F. Duggan Smith. The Driving Skills Book: How to be a smarter driver. Shell Answer Series \# 6. Shell Oil Company. 1991 [no longer in print; available online only; cited 5 February 2009]. Available at: http://shellne.com/smdrive.html

${ }^{6}$ Sir Isaac Newton: The Universal Law of Gravitation. Department Physics \& Astronomy University of Tennessee Cited 9 March 2009. Available online at: http://csep10.phys.utk.edu/astr161/lect/history/newtongrav.html
}

${ }^{7}$ How People Learn: Brain, Mind, Experience, and School. Commission on Behavioral and Social Sciences and Education (CBASSE). 1999 [cited 5 February 2009]. Page 19. Available online at:

http://www.nap.edu/openbook.php?record_id=6160\&page $=19$

${ }^{8}$ Ibid.

${ }^{9}$ Bill Quirk. The NCTM Calls it "Learning Math". Chapter 4. The Truth About Math Standards and Math

Reform. 2006 [cited 5 February 2009]. Available at: http://www.wgquirk.com/chap4.html\#Lessismore

10 Bruce Devlin. "How do we learn math?" March 2006. Article in the monthly online column Devlin's Angle. The Mathematical Association of America. March 2006 [cited 5 February 2009]. Available at: http://www.maa.org/devlin/devlin_03_06.html 